\title{
Norois
}

Environnement, aménagement, société

$241 \mid 2016$

Innovation sociale et développement des territoires dans les campagnes européennes

\section{Le foncier agricole à l'épreuve de la multifonctionnalité : desseins environnementaux et alimentaires dans les métropoles lilloise et nantaise}

Agricultural land in the face of multifunctionality: environmental and nutritional endeavors in the Lille and Nantes metropolitan areas

Christine Margetic, Nicolas Rouget et Guillaume Schmitt

\section{OpenEdition}

Édition électronique

URL : https://journals.openedition.org/norois/6012

DOI : $10.4000 /$ norois. 6012

ISBN : 978-2-7535-5504-4

ISSN : $1760-8546$

Éditeur

Presses universitaires de Rennes

Édition imprimée

Date de publication : 30 décembre 2016

Pagination : 87-104

ISBN : 978-2-7535-5483-2

ISSN : 0029-182X

\section{Référence électronique}

Christine Margetic, Nicolas Rouget et Guillaume Schmitt, « Le foncier agricole à l'épreuve de la multifonctionnalité : desseins environnementaux et alimentaires dans les métropoles lilloise et nantaise », Norois [En ligne], 241 | 2016, mis en ligne le 31 décembre 2018, consulté le 14 janvier 2022. URL : http://journals.openedition.org/norois/6012 ; DOI : https://doi.org/10.4000/norois.6012 


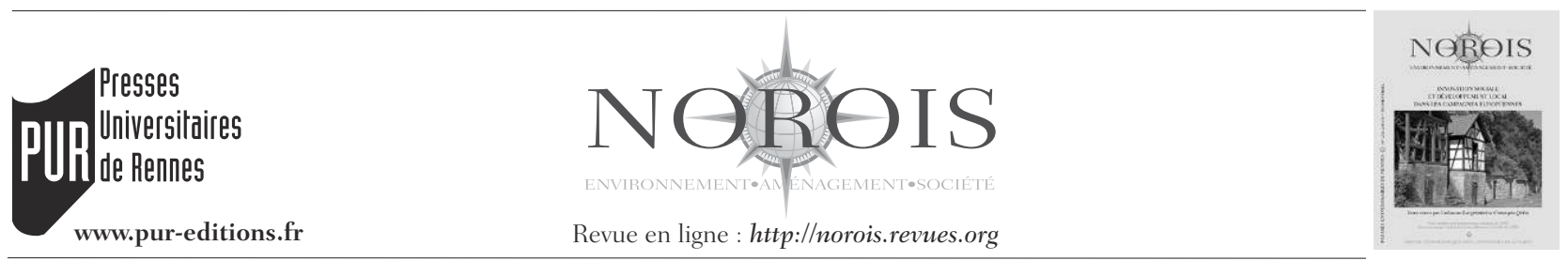

\title{
Le foncier agricole à l'épreuve de la multifonctionnalité : desseins environnementaux et alimentaires dans les métropoles lilloise et nantaise
}

\author{
Agricultural Land in the Face of Multifunctionality: \\ Environmental and Nutritional Endeavors in the Lille and Nantes Metropolitan Areas
}

\author{
Christine Margetic $^{* a}$ Nicolas Rouget ${ }^{b}$ et Guillaume Schmitt $^{b}$
}

\footnotetext{
*Auteur correspondant: Tel. : 0652179693

${ }^{a}$ ESO - UMR CNRS 6590, Université de Nantes, Bât. Igarun - BP 81227, 44312 Nantes cedex 3, France. (christine.margetic@univ-nantes.fr)

b CALHISTE, EA 4343, UVHC, Campus Mont-Houy - 59313 VAlenciennes Cedex 9. (Nicolas.Rouget@univvalenciennes.fr)(schmitt_guillaume@yahoo.fr)
}

Résumé : Comment est donné à lire le foncier agricole dans les métropoles lilloise et nantaise ? Cette question très simple au premier abord est pourtant plurielle, puisque les réponses relèvent, entre autres, du registre concret des systèmes de régulation foncière et d'une recherche de cohérence territoriale pour des usages multiples du sol. Le «faire territoire » ensemble à partir d'une diversité de pratiques interroge alors, notamment en termes d'instances de réflexion et d'action collective en milieux urbains et périurbains. Dans ce contexte, notre objectif est de mettre en évidence comment le foncier agricole se trouve de plus en plus mobilisé dans les projets de ville à travers une entrée paysagère et sa projection idéelle, et plus récemment à travers une entrée alimentaire au nom d'un lien ville/agriculture à restituer. Ainsi, après avoir mis en lumière la mobilisation du foncier en tant que ressource territoriale, nous exposons l'évolution de l'intégration du foncier agricole dans les logiques néo-naturalistes et nourricières des métropoles lilloise et nantaise. Les cas du projet de zone maraîchère sur Lille et de reconquête des friches nantaises nous permettent d'établir la temporalité des projets et de décrypter les logiques d'acteurs sous-jacentes à la surqualification constatée du foncier agricole.

\begin{abstract}
How is agricultural land taken into consideration in the Lille and Nantes metropolitan areas? This apparently very simple question in fact has multiple facets, with answers that relate, for example, to land-tenure regulation systems and efforts to achieve territorial coherence in the face of multiple land uses. Sharing a territory among several concurrent practices raises questions in terms of forums for discussion and collective action in urban and peri-urban areas.

In this context, our aim is to highlight the way in which agricultural land is increasingly involved in urban projects in terms of landscaperelated elements (and the ideal projection of such elements) and, more recently, from a nutritional standpoint in a bid to renew links between the city and agriculture. After underscoring the utilization of agricultural land as a territorial resource, we shall go on to present the changing ways in which agricultural land has been integrated into the neo-naturalist and nutritional goals of the Lille and Nantes metropolitan areas. The cases of a proposed market-gardening zone near Lille and reclaimed wasteland near Nantes shall then enable us to contextualize the time frames of these projects and understand the motivations, on the part of actors and stakeholders that underlie the prolific uses of agricultural land that have been observed.
\end{abstract}

Mots clés : foncier - ressources - durabilité - alimentation durable - Lille - Nantes

Keywords: land - ressources - sustainability - sustainable food - Lille - Nantes 


\section{INTRODUCTION}

Comment est donné à lire le foncier agricole dans les métropoles lilloise et nantaise? Cette question très simple au premier abord est pourtant plurielle. Les réponses relèvent entre autres du registre concret de systèmes de régulation foncière et d'une recherche de cohérence territoriale pour des usages multiples du sol (Bertrand, 2013). Support à des activités dotées de valeurs vénales et/ou symboliques, la terre agricole constitue une interface particulière entre praticiens et lieu, entre processus de territorialisation et action collective (Croix, 1999). Ainsi, qu'elles visent l'extension d'emprises bâties préexistantes ou la patrimonialisation d'espaces ouverts, les stratégies de développement ou d'aménagement conditionnent et sont conditionnées par sa la mutabilité de la terre agricole, sa disponibilité et le marché qui en découle. En fait, bien que propriétés privées, les espaces agricoles participent d'enjeux d'intérêt général et peuvent en cela être érigés en bien commun (Banos et Sabatier, 2010; Poulot, 2013). Cet objet-ressource est une clé d'entrée pour actualiser des valeurs portées par une diversité d'acteurs ayant des objectifs, des pratiques et des appuis territoriaux différents, dont l'approche collective reflète une proximité parfois instrumentalisée (Margetic et al., 2016).

Convoquer les métropoles lilloise et nantaise ne tient pas à une similitude démographique (Lille métropole est deux fois plus peuplée que Nantes métropole), ni à l'équivalence de leur poids agricole (1 099 exploitations à Nantes contre 775 à Lille au RGA de 2010). Et, bien que leur statut administratif ait pris le même tournant en janvier 2015, il est le fruit d'un parcours différent ${ }^{1}$. Pour autant, la « naturalité » des démarches comparatives (Guéranger, 2012) contribue à les relier au travers d'un foncier agricole mobilisé pour sa portée symbolique ${ }^{2}$, à des fins performatives ou concurrentielles et comme outil de gouvernement (Douay, 2013). Les contextes institutionnels amènent à formuler l'hypothèse que

1. Reconnues métropole au titre de la loi MAPAM, Nantes Métropole avait le statut de communauté urbaine depuis 2001 pour 24 communes, quand ce statut datait de 1968 pour Lille Métropole Communauté Urbaine (LMCU) pour 85 communes (devenue la MEL : Métropole Européenne de Lille).

2. Dans les études diagnostics du SCoT de la métropole lilloise (le conseil syndical s'est réuni pour arrêter le SCoT le 26 février 2016), celle-ci est régulièrement présentée comme la métropole « la plus agricole de France ». Cette expression est aussi reprise dans les délibérations communautaires à l'exemple de la délibération du conseil du 15 novembre 2013. ce foncier pensé en bien multifonctionnel est, en quelque sorte, «surqualifié » par surimposition d'attributs. Dans le cadre d'une dynamique d'appropriation par les collectivités locales des problématiques propres au tournant territorial (Pecqueur, 2006), la démarche comparative qui est la nôtre a pour base des travaux antérieurs (Margetic et Chaucesse, 2012 ; Rouget, 2008 et 2012 ; Schmitt, 2009), réactualisés de 2014 à 2016 par une série d'entretiens complémentaires.

Notre objectif est de cerner comment le foncier agricole se trouve explicitement mobilisé dans les projets de ville (Banzo et Couderchet, 2013) à travers une entrée paysagère et sa projection idéelle, et plus récemment, une entrée alimentaire au nom d'un lien ville/agriculture à restituer. Après avoir assis la notion de ressource territoriale (croisée avec les paradigmes relatifs à la question du foncier agricole) et nos terrains d'étude, nous exposerons deux démarches emblématiques de ce processus de « qualification », dont nous questionnerons les fondements réflexifs locaux dans une dernière partie.

\section{LE FONCIER AGRICOLE, UNE RESSOURCE TERRITORIALE EN AFFIRMATION}

Nouvelle frontière agraire pour certains, urbaine pour d'autres, le renouvellement des liens ville/nature/ agriculture oblige les acteurs à explorer des voies d'articulation entre des intérêts contradictoires dans des territoires en construction (Loudiyi et al., 2011). Le foncier est érigé en clef de voûte de projets de territoire dans de nombreux guides méthodologiques à destination des acteurs de l'aménagement et de la planification de l'espace (par exemple: DGUHC, 2004). Ainsi, après avoir suscité un moindre intérêt durant les années 1960 et 1970, l'enjeu foncier émerge à nouveau depuis une quinzaine d'années. Relatif aux modalités d'urbanisation à l'œuvre autour des agglomérations, ce renouveau est à corréler au processus de dilatation urbaine (Schmitt, 2009). Dans cette perspective, le foncier agricole est envisagé comme une ressource territoriale susceptible de caractériser un territoire spécifique et ce, dans une optique de développement (Glon et Pecqueur, 2006), notamment en zone périurbaine (Bertrand, 2013).

Les valeurs attribuées au foncier agricole, conformément à la grille d'identification d'une ressource 
territoriale $^{3}$ de Pecqueur et al. (2012), ont évolué au fil du temps, en réponse à des enjeux politiques (un paradigme agro-industriel reflet de choix opérés dans le cadre de la Politique agricole commune) ou sociétaux (intégration de questionnements environnementaux pour le paradigme néo-naturaliste) eux-mêmes évolutifs (tableau 1). Mettant en relief la grande cohérence des différentes conceptions (Lévy, 2010), cette classification aide à comprendre la complexité à élaborer un projet collectif agricole (Bertrand, 2013).

Le paradigme post-matérialiste qui s'affirme à partir des années 2000 donne à lire cette complexité. L'éthique de la durabilité intègre des attentes sociétales formulées en termes de "désirs » de la part d'habitants (agriculteurs ou non, visiteurs) s'appropriant ces lieux (Le Caro et al., 2010). Il en découle une projection idéelle, parfois reflet d'une vision «passéiste » (paradigme agraire) ou intemporelle (paradigme néo-naturaliste), qui s'affranchit souvent de la phase de modernisation agricole productiviste (paradigme agro-industriel). Convoqué d'abord pour légitimer ou assurer la préservation d'espaces de nature, le foncier agricole est désormais aussi sollicité pour une finalité alimentaire. Résultant de choix volontaristes de la part d'acteurs pourvus d'intentionnalités marquées (Pecqueur et al., 2012), ces orientations sont diversement repérables dans les projets des métropoles françaises (Brand et Bonnefoy, 2011).

Outil de médiation autour d'enjeux nature/alimentation, la mobilisation du foncier agricole s'avère pourtant le moyen d'expérimenter des solutions adaptatives originales. Patrimonial (paradigme agraire), substituable (paradigme agro-industriel), ce bien public «immuable» (le long terme dans l'échelle de temps du paradigme néo-naturaliste) s'offre «mutable» (plusieurs fonctions sur une

\begin{tabular}{|c|c|c|c|c|}
\hline & \multicolumn{4}{|c|}{ Paradigme en débat } \\
\hline & Agraire & Agro-industriel & Néo-naturaliste & Post-matérialiste \\
\hline $\begin{array}{l}\text { Place du foncier agri- } \\
\text { cole (FA) }\end{array}$ & $\begin{array}{l}\text { Objet agronomique } \\
\text { Ressource disponible } \\
\text { pour une alimentation } \\
\text { de proximité }\end{array}$ & $\begin{array}{l}\text { Objet-moyen d'action. } \\
\text { Ressource disponible } \\
\text { pour une transformation } \\
\text { économique }\end{array}$ & $\begin{array}{l}\text { Acteur dans l'action. } \\
\text { Valeurs intrinsèques, } \\
\text { source de droits et de } \\
\text { devoirs pour l'Homme }\end{array}$ & $\begin{array}{l}\text { Composante de l'action. } \\
\text { Patrimoine historique- } \\
\text { ment construit et bien } \\
\text { public à gérer et à pro- } \\
\text { téger collectivement }\end{array}$ \\
\hline Type de dessein & Auto-centré & Consommation & Préservation & Conciliation \\
\hline $\begin{array}{l}\text { Relation développement } \\
\text { / mobilisation foncière }\end{array}$ & Conjoncture & Opportunité & Conservation & Projection « idéelle» \\
\hline Système de valeurs & $\begin{array}{l}\text { Orienté sur la produc- } \\
\text { tion }\end{array}$ & $\begin{array}{l}\text { Orienté sur la } \\
\text { productivité }\end{array}$ & Fondé sur la culpabilité & Éthique de la durabilité \\
\hline $\begin{array}{l}\text { Logique du système } \\
\text { productif }\end{array}$ & Alimentaire & Économique & Environnementale & Territoriale \\
\hline $\begin{array}{l}\text { Ressort de l'activité } \\
\text { productive }\end{array}$ & Nécessités & $\begin{array}{l}\text { Demande: } \\
\text { programmation, } \\
\text { standardisation }\end{array}$ & $\begin{array}{l}\text { Besoins : } \\
\text { tradition, adaptation }\end{array}$ & $\begin{array}{l}\text { Désirs : } \\
\text { co-construction, } \\
\text { innovation }\end{array}$ \\
\hline Acteurs dominants & $\begin{array}{l}\text { Propriétaires, } \\
\text { exploitants }\end{array}$ & $\begin{array}{l}\text { Agents économiques, } \\
\text { professionnels, élus }\end{array}$ & Communautés & Individus/collectifs \\
\hline Echelle de temps & Court et long termes & Court et moyen termes & Long terme & $\begin{array}{l}\text { Le temps au centre des } \\
\text { questions foncières }\end{array}$ \\
\hline $\begin{array}{l}\text { Valeurs liées au FA: } \\
\text { société }\end{array}$ & Bien-fonds & Bien mobile & Bien « naturel » & Bien multifonctionnel \\
\hline $\begin{array}{l}\text { Valeurs liées au FA: } \\
\text { monde politique }\end{array}$ & $\begin{array}{l}\text { Patrimonialité } \\
\text { (entre soi familial) }\end{array}$ & $\begin{array}{l}\text { Substituabilité } \\
\text { (entre soi agricole) }\end{array}$ & $\begin{array}{l}\text { Immuabilité } \\
\text { (entre soi idéologique) }\end{array}$ & $\begin{array}{l}\text { Mutabilités } \\
\text { (multi-acteurs) }\end{array}$ \\
\hline
\end{tabular}

3. La grille repose sur des critères de qualification de la coordination collective, d'appropriation commune, d'apprentissage cumulatif, de spécification progressive et de territorialisation instituée.
Tableau 1: Le foncier agricole en tant que ressource territoriale (source : d'après Lévy [2010] - réalisation : Margetic, Rouget, Schmitt [octobre 2015])

Agricultural Land as a territorial resource 
même parcelle dans le cadre du paradigme postmatérialiste). Au regard des critères d'identification d'une ressource territoriale, sa mise en vitrine en tant que bien multifonctionnel témoigne d'une appropriation multi-acteurs dans un contexte de territorialisation renouvelée des logiques agricoles. Serge Bonnefoy (2011) démontre un enracinement des politiques agricoles d'agglomération qui combinent une certaine standardisation à une diversité propre à l'émergence de nouvelles formes d'agriculture. Même si, juridiquement, la compétence « agriculture » n'existe pas, les métropoles se sont emparées de la question agricole, et revendiquent un rôle dans l'orientation de l'agriculture locale. Pour y parvenir, un moyen possible est d'interagir à partir du foncier.

\section{L'AGRIURBAIN POUR HORIZON}

Ériger le foncier agricole en ressource territoriale oblige à repérer des invariants dans les itinéraires des métropoles lilloise et nantaise. Après avoir explicité notre méthodologie, l'analyse parallèle des trajectoires des deux territoires permettra de cerner la capacité des acteurs locaux à co-construire des référentiels fonciers emprunts de nouveauté.

\section{Interroger les convergences et les modalités de mise en ouvre de projets territoriaux agriurbains ${ }^{4}$}

La démarche méthodologique combine deux volets. Dans un premier temps, nous avons croisé différents travaux déjà conduits par Margetic sur Lille (Chantier Nature, 1999) et Nantes (Margetic et Chaucesse, 2012) $)^{5}$ d'une part, par Rouget (2008 et 2012) et Schmitt (2009) sur Lille d'autre part. L'article se fonde ainsi sur des entretiens semidirectifs auprès d'agriculteurs, d'élus et agents des Chambres d'agriculture départementales (CAgr.) et d'instances urbaines (mairies, Communautés urbaines), sur des travaux d'analyse des documents d'urbanisme successifs, et d'entretiens plus infor-

4. Au regard du nombre de publications sur ce sujet depuis le début des années 2010, nous n'avons retenu pour la suite de l'article que les publications ayant trait à nos terrains d'étude. De plus, le suivi depuis une vingtaine d'années pour Lille et une dizaine d'années pour Nantes nous fournit un corpus important et disparate que nous avons retravaillé au regard de la problématique posée.

5. Jean-Noël Chaucesse est le responsable du service Territoires de la Chambre d'agriculture de Loire-Atlantique. mels au cours de journées sur l'agriculture périurbaine qui se tiennent localement depuis le milieu des années 2000.

Pour conforter l'approche comparative et valider notre grille réflexive, nous avons entrepris des recherches complémentaires pour une mise en visà-vis des regards portés par les acteurs de la planification dans chacun des terrains. Des entretiens semi-directifs (non enregistrés) ont été réalisés en 2015 et 2016 sur Lille ${ }^{6}$ et Nantes ${ }^{7}$. L'objectif était de repérer les convergences et divergences stratégiques des politiques conduites localement pour pouvoir en analyser les ressorts au-delà des seules ambitions générales.

\section{Des « natures » et des acteurs}

Le désir de nature en ville s'exprime par une diversité d'images, de formes, d'expériences. Les espaces de nature sont pluriels (Banzo et Couderchet, 2013). Espaces enchevêtrés et insérés jusque dans le tissu urbain sous forme d'espaces agricoles ou boisés, ils comprennent également une nature plus sauvage, des zones humides, des friches, des délaissés, autant que des espaces très aménagés (jardins publics et privés, parcs urbains - Novarina, 2003). Les espaces de nature cultivée, supports de fonctionnalités multiples et d'usages imbriqués, sont complexes à appréhender, alors qu'ils sont concernés par des politiques d'aménagement aux structures préétablies qui les considèrent souvent selon une seule fonctionnalité, et non en tant que bien multifonctionnel (tableau 1).

Le fait de traiter le foncier agricole par la nature dans une vision d'ensemble constitue de fait une nouvelle question, qui impose de réajuster la gouvernance relativement aux visions fonctionnelles ou idéelles d'acteurs toujours plus nombreux : agriculteurs, représentants du monde agricole, aménageurs, membres d'associations, collectifs d'usagers-habitants, citadins et bien évidemment élus (Bertrand, 2013). Les discours et les pratiques s'appuient sur

6. Le vice-président à l'espace rural et à l'économie agricole de la métropole de Lille, les chargés de mission du service Développement économique et du service Aménagement et habitat respectivement responsables de la filière agriculture et référent des questions agricoles à la sous-direction du foncier, ou encore des élus municipaux (Vendeville notamment - « plaine » des Périseaux).

7. Les deux vice-présidents successifs à NM, 8 élus, 5 agriculteurs, les chargés de mission de la Chambre d'agriculture, de CAP44 et l'association AIRES. 
des contextes différents à Lille et à Nantes (figure 1 - planche I).

\section{À Lille, du vert à l'agricole...}

Deux documents d'urbanisme - le Schéma directeur d'aménagement et d'urbanisme (SDAU) de 1973 et le Schéma directeur (SD) de 2002 reflètent les choix stratégiques et les rapports de force existant au sein de la métropole lilloise quant à la question agricole.

Des années 1970 aux années 1990, si l'intégration des « espaces naturels » dans le projet de territoire a pu être d'emblée unanimement actée dans le contexte d'une politique de rattrapage du «vert » (Schmitt, 2007), celle de l'activité et des espaces agricoles ne relevait aucunement de l'évidence. Les projets ont été positionnés aux dépens de, parfois en opposition à l'agriculture. Ainsi, en vue de créer une zone de loisirs s'étirant sur $16 \mathrm{~km}$ au sud-ouest de la conurbation, le SDAU validait le premier projet de parc de la Deûle sur 1200 ha de terres agricoles. Bruno Bonduelle (1992), grande figure patronale lilloise impliquée dans la gouvernance locale, suggérait même d'offrir aux agriculteurs deux fois l'indemnité couramment pratiquée pour transformer le cadre de vie des citadins en consacrant 20000 nouveaux ha à l'habitat individuel et à la forêt. De son point de vue, les terres agricoles n'étaient pas prioritaires.

À l'inverse et dans les faits, la reconnaissance urbaine d'une agriculture contribuant au projet métropolitain s'est opérée au cours des 13 années (1989-2002) de révision du SD (Rouget, 2014). Celui-ci ambitionnait d'atteindre en 2015 « 10000 hectares voués à la nature et aux loisirs » (p. 94) dont « 3000 devant conserver leur usage agricole » (p. 191) et devant remplir « une double fonction » (p. 196). Ce compromis résulte en particulier de la fermeté de la Chambre d'agriculture, qui menace les élus du Syndicat mixte du SD d'un recours au tribunal administratif pour vice de forme (Serrano, 2012). Il entérine néanmoins la promotion d'une campagne simultanément cadre paysager et lieu de production, qui intègre à ses stratégies de développement la publicisation de cet espace. La progression dans la lecture respective de ce foncier a permis un point de convergence des intérêts entre élus, aménageurs et acteurs du monde agricole. Ainsi, Bernard Pruvot, président de la CAgr., concède en 2002 : "La vocation de l'agriculture est de nourrir les Hommes. Néanmoins, nous devons engendrer une mutation qui préserve des espaces de transformation des produits agricoles et de services. " Directeur du syndicat mixte Espace Naturel Lille Métropole - $\mathrm{ENLM}^{8}$, Pierre Dhénin signifie aux agriculteurs de l'est lillois que, "l'objectif du syndicat mixte est celui d'un paysage vivant, ouvert à la promenade et économiquement faisable, donc en collaboration avec les agriculteurs » (janvier 2005, réunion d'information); agriculteurs qui considèrent alors qu' «il y a une culture du positif à faire » : "Quoi qu'il en soit, on doit entretenir..., autant aller au-delà pour participer au bien commun» (entretiens, 2005).

\section{...Et du vert agricole à Nantes}

À la différence du contexte lillois, les liens sont moins conflictuels entre Nantes métropole (NM), CAgr et l'Agence d'urbanisme de Nantes (AURAN) ${ }^{9}$. Cela repose d'une part, sur le fait que la CAgr. était présidée jusqu'en 2007 par la Confédération paysanne, qui porte une vision ruraliste selon l'un des adhérents, et d'autre part, sur la qualité de liens interpersonnels, inscrits dans la durée. En effet, Jean-Pierre Legendre a été VicePrésident (VP) chargé de l'agriculture urbaine à NM de 2001 à 2014, quand Jean-Noël Chaucesse (Chambre d'agriculture) et Bruno Julien (AURAN) suivent la question agricole depuis une trentaine d'années. Aussi, dans le contexte d'orientations agricoles diverses (élevage laitier et bovin, viticulture, maraîchage...), un enjeu transversal émerge dès les années 1970 : l'intégration de la problématique périurbaine pour préserver une agriculture vivante plus ou moins productiviste. Par manque de relais politique, toutes les démarches impulsées sont alors restées d'ordre théorique, même si des initiatives sont à souligner (programme Pollen à Bouguenais en 1994 qui donne naissance au réseau «Villes fertiles » associant 7 villes du sud-ouest nantais, la Chambre d'agriculture et l'AURAN en 1996) (entretien avec M. le maire).

\footnotetext{
8. Le syndicat mixte associe alors 40 communes et l'EPCI. Les objectifs assignés à l'ENML sont, notamment, de répondre aux besoins de nature et d'espaces de proximité, d'améliorer la qualité paysagère, de protéger, gérer et restaurer les sites naturels à haute valeur écologique...

9. Selon un responsable de la CAgr., "l'AURAN est notre support! C'est elle qui se positionne comme l'interlocuteur sur la question de l'agriculture périurbaine».
} 
Dès 2000, l'appropriation politique passe par la signature d'un protocole de partenariat ${ }^{10}$ entre le District et la CAgr. en vue de la création de l'association Terres en Villes, qui prévoit un soutien technique et financier aux « territoires agricoles durables » et aux « zones naturelles interstitielles » proches d'espaces urbanisés, peu propices à une agriculture pérenne. Si d'autres conventions suivent, l'approbation du SCoT Nantes-Saint-Nazaire en juin 2003 acte la réalisation d'une carte de l'agriculture pérenne, avec identification des zones de fragilité donnant lieu à une vigilance particulière, comme les espaces de prés marais (entretien AURAN).

Ainsi, on peut parler d'une éthique de la durabilité dans le raisonnement suivi par les trois acteurs-clés que sont la Chambre d'agriculture, NM et l'AURAN. Espaces agricoles et naturels sont pensés en concordance, et l'interconnaissance est réelle grâce à la réalisation régulière de diagnostics agricoles (7 en 30 ans!) et d'une cartographie associée. Réalisés par la CAgr. et financés par NM, les derniers diagnostics montrent une poursuite de l'érosion du nombre des exploitations (-28\% entre 2004 et 2012), ce qui conforte le choix politique porté par Jean-Pierre Legendre de privilégier les installations lorsque c'est envisageable, notamment pour les 9500 ha de zones humides classés dans les PLU (entretien, 2014). Est ainsi encouragé le pâturage extensif qui nécessite de vastes surfaces, en particulier en rive sud de la Loire (Margetic, 2014).

Du vert avant l'agricole sur Lille, au vert parallèlement à l'agricole sur Nantes, ces métropoles sont à l'affût de «bonnes pratiques » reproductibles pour un maintien des espaces agricoles, et exemplaires par leur dimension multifonctionnelle. L'originalité des expériences plus récentes tient au volet « relocalisation de la production alimentaire » qui devient central.

\section{EXPÉRIMENTER EN TERRES AGRICOLES URBAINES ET PÉRIURBAINES}

À partir des années 2000, les projets d'intégration fonctionnelle des espaces agricoles et de l'agriculture à la ville privilégient la concertation dans le cadre de politiques emblématiques qui donnent lieu à des

10. « Pour le développement d'une agriculture innovante dans l'agglomération nantaise ». échanges. Les métropoles de Lille et de Nantes n'y dérogent pas, et participent à différentes instances de mise en commun d'expériences, telle Terres en Villes. Relevant d'objectifs souvent proches, celles-ci sont redevables des contextes politiques, agricoles et environnementaux locaux.

\section{À Lille : des expériences plurielles}

Pour Vidal et Fleury (2009), toute « métropole verte » doit structurer une politique de soutien à l'essor de l'agriculture biologique. La métropole de Lille signe une convention en 2009 avec le Groupement des Agriculteurs Biologiques du NordPas-de-Calais (GABNOR), afin d'accompagner la transition vers ce mode de production. L'objectif est alors de protéger les champs captant au sud du territoire et de conforter l'approvisionnement bio dans la restauration collective. Depuis, la métropole s'est concrètement engagée (2013) dans la création d'une zone maraîchère et horticole localisée au sudouest de l'agglomération sur le «site de la Vallée » à Wavrin (figure 2 - planche II). Positionné sur des réserves foncières initialement constituées pour l'élargissement de la RN 41, le regroupement des parcelles est le fait du remembrement consécutif au projet d'aménagement routier. À la différence des «domaines agricoles » constitués en région parisienne dans les années 1970 pour compensation de pertes foncières (Darly, 2012), la zone de Wavrin reflète une démarche de soutien au développement d'exploitations nouvelles, fondée sur une stratégie d'ancrage de l'activité dans des systèmes de production (maraîchage, arboriculture) et des itinéraires techniques (agriculture biologique, ou à défaut, certification environnementale de niveau $\left.3^{11}\right)$ conformes à l'éthique de la durabilité. Ce choix s'explique tant par la proximité d'une zone de captage d'eau, que par la volonté de répondre aux aspirations citadines les plus actuelles (nourrir la ville, préserver les ressources...).

Ce projet est original à plusieurs titres. Tout d'abord, son ampleur. À terme, il pourrait couvrir

11. Le décret $n^{\circ} 2011-694$ du 20 juin 2011 du code rural relatif à la certification environnementale des exploitations agricoles répond au besoin clairement exprimé dans les travaux du Grenelle de l'environnement. Déclinée en 3 niveaux, le plus élevé est qualifié de «Haute Valeur Environnementale », et est fondé sur des indicateurs de résultats relatifs à la biodiversité, la stratégie phytosanitaire, la gestion de la fertilisation et de l'irrigation. 
47 ha mis en réserve à la SAFER à la demande de la métropole de Lille. De plus, cet espace est équipé (construction d'un hangar modulable, réalisation d'un forage et d'un réseau d'irrigation) et il est prévu que soit mise en place une structure de mutualisation du matériel agricole.

Ensuite, la diversité des profils des porteurs de projet et les productions envisagées. À la suite d'un appel à candidature lancé en 2013 ${ }^{12}, 8$ candidats (sur 25 dossiers dont 24 en bio) sont retenus, depuis l'ouvrier agricole qui s'installe à son compte, au jeune producteur qui voit ici le moyen de délocaliser et d'agrandir son exploitation, jusqu'à l'agent d'assurance de 50 ans en reconversion professionnelle. Tous partent en Agriculture Biologique, sur du maraîchage (3 dossiers), de l'arboriculture (2), des fruits rouges et des plantes aromatiques (2).

Zone « vitrine » pour la Métropole et dans une certaine mesure pour la profession agricole, ce projet renvoie à une politique de filière, non sans décalage avec les expérimentations conduites à compter de la fin des années 1990 au sein des «parcs périurbains » (parcs du Val de Marque et de la Deûle notamment) dessinés par le syndicat mixte ENLM (figure $3 \mathrm{a}$ - planche II). Prenant appui sur les espaces agricoles répertoriés dans le SD de 2002, une politique partenariale est alors proposée à l'ensemble des agriculteurs autour de deux axes : un programme d'intégration paysagère (plantations de haies, fossés végétalisés) et un programme de mise en œuvre de circuits de fermes (opération de promotion des ateliers de diversification agricole). Toutes ces opérations bénéficient d'outils de communication, soit pour valoriser les opérations d'envergure, sous forme de grands panneaux informatifs (figure 3 - planche II), soit par des dépliants diffusés par les équipements ENLM et les offices du tourisme. L'enjeu est de produire du paysage, mais aussi d'accompagner les trajectoires agricoles pour une territorialisation et une ouverture de l'activité et de ses espaces aux citadins (Rouget, 2012).

\section{Nantes : reconquérir les friches agricoles}

En Loire-Atlantique aussi, la question des terres agricoles interpelle les instances, mais sous une autre forme. Apparues dans les années 1970, les

12. Consultable sur [http://www.lillemetropole.fr]. friches de déprise, d'abandon vont gagner le nord de la Loire après s'être cantonnées au sud. Cette diffusion est facilitée par l'absence de cadre juridique prescriptif (hormis le POS), le SDAU n'ayant pas été mené à terme par les élus de l'Assemblée Communautaire de la Région Nantaise au début de ces années (Geniaux et Napoléone, 2007). Pour autant, les friches agricoles sont perçues comme un échec social. Les élus sentent qu’ils doivent se mobiliser pour leur rendre de la valeur (Le Caro et al., 2010), mais sans vraiment agir avant la fin des années 1990.

Ainsi, les premières actions volontaristes en nord Loire émanent du monde agricole, d'un groupe d'agriculteurs de La Chapelle-sur-Erdre et de Treillières (nord de Nantes). Dès 1994, ils créent une Association Foncière Agricole (AFA) ${ }^{13}$, qui devient la Société civile d'exploitation agricole Nord-Loire en intégrant la commune d'Héric, avant de devenir la Société coopérative d'intérêt collectif (SCIC) NordLoire en 2013. Pour l'un des agriculteurs, les discussions autour de l'aménagement foncier ont pris place dans " un cadre plus serein à partir de juin 2001 », grâce à un "outil de médiation ", "un groupe de travail constitué d'une vingtaine d'agriculteurs, de représentants d'associations et de propriétaires, qui va rédiger la Charte de l'espace rural de La Chapelle-sur-Erdre signée en octobre $2000 »$. En sud Loire, l'innovation vient de la commune de Bouguenais, où dès 1997, l'association Actions initiatives rurales et sylvicoles (AIRES) entend soutenir des projets de maintien et de relance d'une agriculture pérenne face à la disparition des petites fermes de polyculture-viticulture. Les membres du bureau se chargent de rencontrer les propriétaires pour leur faire signer une lettre d'engagement de remise en état des parcelles, et suscitent la création des AFA de la Pierre Anne (1999) et des Ecobuts (2008).

La politique de reconquête des friches agricoles est formulée explicitement pour la première fois dans un protocole signé entre le District et la CAgr. en décembre 1999. Dans ce cadre, le District prend en charge $80 \%$ du coût du défrichage ${ }^{14}$. Elle est confirmée lors des réflexions liées au SCoT : alors

13. Concomitamment, sous l'impulsion de la CAgr., sont créées d'autres AFA : Saint Aubin des Châteaux (1993-1998), Erbray (1995-1998), Le Pellerin (1999) et la plus récente à Saint-Jean-de-Boiseau (2012).

14. Pour les $20 \%$ à charge des propriétaires, certaines communes investissent, comme Saint-Léger-les-Vignes qui finance la moitié des montants dus (Margetic, 2014). 
que la vocation agricole est assurée pour 15900 ha, les communes et la CAgr. décident collectivement de bâtir une stratégie pour gagner en surfaces, en ciblant de manière originale la remise en culture des friches à grande échelle. Le programme d'actions concertées se déroule en trois phases (Margetic et Chaucesse, 2012).

De 2007 à 2009, dans la phase 1, avec l'appui financier de NM, la CAgr. a réalisé un diagnostic sur les 28000 ha de zones Agricoles et Naturelles des PLU de l'agglomération. L'objectif est de repérer et de rendre lisible la totalité des friches à l'échelle de la parcelle. Le constat est édifiant : 3700 ha de friches de moins de 6 ans, exploitables sur un plan technique après défrichage, et 1500 ha occupés par des chevaux de loisir sur de petites parcelles souvent en location ${ }^{15}$. Au total, 155 sites sont disséminés dans la trame urbaine et agricole (figure 4 planche III). L'objectif opérationnel vise la remise en état de 500 ha d'ici fin 2014, avec au moins un site défriché par commune en 2011 , et 100 projets (20 installations sur des entités de 3 à 10 ha, et 80 exploitations existantes confortées).

Courant 2010, la phase 2 aboutit à la co-construction du programme d'actions par méthode participative, avec implication des initiateurs, des communes, des professionnels du monde agricole pour la validation de secteurs d'intervention prioritaires par commune (de 43 sites alors à 83 en 2013). Comme l'indique le VP de NM : «Compréhension $d u$ territoire, patience, respect des interlocuteurs, telles sont les trois règles de base pour la réussite de ce projet, qui prend forme dans de nouvelles scènes de débat. »

La phase 3 débute en 2011 par un séminaire destiné à mobiliser tous les acteurs du monde rural et agricole, en premier lieu les propriétaires de parcelles. Elle se poursuit actuellement par des opérations concrètes de défrichage et de ré-exploitation des terres et, plus original, par l'organisation d'une journée Agriculture urbaine annuelle (120 personnes en 2016).

En juin 2014, selon les données de NM, la démarche est enclenchée sur 85 sites pour 1165 ha (figure 5-planche IV). Concrètement, les 51 défrichages sur 450 ha ont contribué à 12 installations

15. Les parcelles à chevaux sont clairement pointées car, souvent en état d'enfrichement latent, leur dispersion renforce une déstructuration du parcellaire agricole. La volonté politique est donc d'intégrer leurs propriétaires le plus en amont possible dans la réflexion.
(84 ha), 9 en cours et 9 à organiser. Les résultats peuvent paraître symboliques (7,3\% de la SAU), mais ils reflètent la difficulté d'agir dans un domaine où prédominent le droit du propriétaire, un différentiel du prix du foncier de l'ordre de 1 à 20 (Margetic et Chaucesse, 2012) et, pour certaines communes, dans le cadre de la loi littoral qui interdit toute construction $^{16}$. Les avancées sont d'un autre registre. En effet, les propriétaires sont moins dans une stratégie d'attente. Ils ont souvent intégré « l'obligation $d u$ maintien d'une agriculture périurbaine, ce qui facilite les échanges » (entretien avec le maire de Saint-Jeande-Boiseau). Parallèlement, d'autres limites tiennent en l'exigence d'une mobilisation dans la durée de multiples acteurs (environ 100 réunions/an) et en la faible surface individuelle des terres à défricher (souvent moins de 3 ha, 20 ares en sud Loire).

Au fil du temps, la volonté politique a été suffisamment forte dans les deux terrains pour qu'effectivement aboutissent plusieurs concrétisations innovantes en termes d'identification par la ville d'une agriculture dont les dynamiques sont perçues d'intérêt général. Pour autant, un certain nombre de limites interroge la portée des montages réalisés et la capacité réelle des acteurs à articuler les différentes dimensions (paysagères, environnementales, nourricières) d'un projet agricole et territorial global.

\section{De la mutabilité DU FONCIER AGRICOLE}

Les deux trajectoires lilloise et nantaise mettent en exergue un processus de (sur)qualification du foncier agricole directement corrélé à une convergence d'intérêts plus ou moins précoce entre acteurs agricoles, planificateurs et monde politique. Devenue de fait un outil de médiation autour des enjeux nature/ alimentation, la mobilisation du foncier agricole donne à comprendre les préceptes d'une durabilité revendiquée pour le moins dans le discours.

\section{Des projets autour d'espaces agricoles sur Lille et une politique agricole sur Nantes}

Composante de l'action, le foncier agricole l'est assurément, d'autant que les surfaces tendent à

16. Pour l'adjointe à Bouaye : «d'une certaine manière, cette loi contre-productive conforte les friches!». 
se rétracter dans tous les territoires. Ce constat explique l'intérêt renouvelé de nombreux acteurs pour cet objet-ressource qui fait lien (nature/agriculture, bien-être des citadins/lieu de loisirs...). Construit à partir d'enseignements tirés des terrains lillois et nantais, le tableau 2 met en évidence le fait que les divergences relevées tiennent pour l'essentiel en une appropriation de la question sensiblement différente et en un jeu des acteurs inégal.

La nature des projets, leur ampleur et leurs finalités constituent des différenciations fortes. Sur Lille, les deux grands projets initiés visent à « orienter » le foncier agricole dans le but d'une intégration de l'agriculture à la cité. Si l'outil déployé à l'échelle des 40 communes ENLM entrait par le paysage donc corrélé au paradigme néo-naturaliste - et avait pour objectif la publicisation des espaces agricoles, la conception pour Wavrin est toute autre. La zone n'est pas destinée à "refaire campagne » (Poulot et Rouyrès, 2007) : elle n'est pas ouverte au public bien que localisée sur un axe majeur de migrations pendulaires (pas de structure d'accueil, aucun cheminement, ni de dispositions paysagères). Alors que les parcs périurbains devaient composer avec un tissu agricole préexistant, la création du « site de la Vallée » s'avère une opportunité localisée dont l'ob- jet-support est substituable au regard des choix de production (et peut en cela être rapprochée partiellement d'une logique qui serait celle du paradigme agro-industriel). Pour autant, propriétaire du foncier, la métropole de Lille s'est résolument engagée dans une démarche multi-partenariale ${ }^{17}$, source d'un volet original : l'installation sur 4 ha d'un espace-test agricole (pépinière de maraichage ou incubateur) à la demande de la Région. Cette orientation renvoie bien à la co-construction d'un bien public à enjeu collectif.

Sur Nantes, la démarche est plus intégrante dès la conception de la politique de valorisation des friches. On repère des éléments du paradigme agraire (bien agronomique), néo-naturaliste (des espaces de biodiversité) et agro-industriel (répondre à une demande d'alimentation locale). Appréhendées en tant qu'espaces mutables en devenir, les friches dans l'espace métropolitain servent d'ancrage à un projet agricole plus global. En effet, la conception d'une méthode empirique et normée à la fois vise une appropriation rapide qui se matérialise par des choix en termes de conduite du projet. Si la politique d'ensemble est définie conjointement par NM et la CAgr., concrètement, chaque commune est systématiquement impliquée (élu(e) et/ou chargé de

\begin{tabular}{|c|c|c|c|}
\hline & \multicolumn{2}{|c|}{ Lille } & Nantes \\
\hline Type de projet & \multicolumn{2}{|c|}{ Foncier agricole à orienter } & Foncier agricole à reconquérir \\
\hline Période & Années 1990-2000 & Années 2010 & mi-2005 \\
\hline Lieu & $\begin{array}{l}\text { Parcs de la Deûle et du Val } \\
\text { de Marque }\end{array}$ & Wavrin & Parcelles identifiées \\
\hline Finalité & $\begin{array}{l}\text { Paysages et promotion d'une } \\
\text { agriculture urbaine }\end{array}$ & $\begin{array}{l}\text { Soutien à une filière marâ̂- } \\
\text { chère et nourrir la ville }\end{array}$ & Promouvoir une agriculture pérenne \\
\hline Outil & Parc périurbain & Zone maraîchère & Politique communautaire \\
\hline Procédure & Contractualisation & Appel à projets & Démarche partenariale et gouvernance locale \\
\hline Modalités & Partenariat / programmes & Location de foncier équipé & $\begin{array}{l}\text { Remise en culture de terres pour des exploita- } \\
\text { tions nouvelles ou confortées }\end{array}$ \\
\hline Jeux d'acteurs & ENLM & Métropole de Lille & NM/CA/élus/associations/agriculteurs \\
\hline Territoire d'action & $\begin{array}{l}\text { Les } 40 \text { communes membres } \\
\text { d'ENLM }\end{array}$ & $\begin{array}{l}\text { Lotissement agricole- } 47 \text { ha } \\
\text { à terme. }\end{array}$ & Les 24 communes \\
\hline
\end{tabular}

Tableau 2 : «Désirs » de foncier agricole dans les projets des métropoles lilloise et nantaise (réalisation : Margetic, Rouget, Schmitt [octobre 2015])

Potential agricultural land resources in development projects in the Lille and Nantes metropolitan areas
17. Agence de l'eau Artois-Picardie, Chambre d'agriculture de région (CAR), Conseil régional et Conseil départemental, Fédération régionale des coopératives d'utilisation du matériel agricole (FRCUMA), GABNOR, l'Association de Formation et d'Information Pour le développement d'initiatives rurales (AFIP), Terres de Liens, lycées agricoles du secteur, Pôle légumes de la CAR, Société d'aménagement foncier et d'établissement rural (SAFER), Société pour la réalisation et la gestion du marché d'intérêt national de Lille (SOGEMIN). 
mission ${ }^{18}$ dans le déroulé le plus en amont possible (choix des périmètres d'action, hiérarchisation des zones). Puis est constitué un groupe composé des deux initiateurs, des propriétaires (pour susciter la création d'une AFA) ${ }^{19}$ et d'autres acteurs du monde agricole en fonction des spécificités communales ${ }^{20}$, dont la SCIC Nord-Loire.

\section{Des candidatures individuelles pour un enjeu collectif}

Autre point de différenciation : la procédure de sélection des candidats ou repreneurs. Pour Wavrin, afin de susciter des projets professionnels rapidement opérationnels, est lancé un appel à candidatures national, en juillet 2013, puis en janvier 2015. Fait novateur, la rédaction du cahier des charges a été partagée avec l'ensemble de la profession (conventionnel et biologique). En pratique, les candidat(e)s sont sélectionné(e)s puis auditionné(e) s par un comité d'agrément ${ }^{21}$. À l'issue, outre le paiement de loyers pour la location de la parcelle et l'utilisation du bâtiment mutualisé, il est attendu des porteurs de projet qu'ils mutualisent leurs moyens et développent des actions collectives en lien avec les besoins du territoire (la restauration collective par exemple). Depuis septembre 2015, cette mobilisation collective a pris la forme d'une association, «La voix des champs bio des Weppes ». Créée à l'initiative des quatre premiers locataires, son but est d'encourager les initiatives communes.

Sur Nantes, "trouver un repreneur si la parcelle est encore enfrichée n'est pas évident. Les candidats éventuels sont rebutés par l'aspect visuel » selon le chargé de mission de la $\mathrm{CAgr}^{22}$. Aussi, la reprise des terres avant leur défrichage n'est plus privilégiée par NM, mais plutôt un bail à 48 mois avec un «intermédiaire »(SCIC Nord-Loire ou entrepreneur agricole)

18. Le diagnostic réalisé durant le phase 1 en 2008 a permis aux nouveaux maires de mieux appréhender la réalité de leur territoire avec des éléments objectifs qui dépassaient la seule problématique des friches, et à réinvestir la question agricole (entretien avec le VP de NM, maire de Brains).

19. Selon un membre de l'AFA des Ecobuts, ce mode de faire est largement plébiscité par les propriétaires parfois démunis face à un bien « qui les encombre » (entretien, 2015).

20. Par exemple à Bouguenais, le Groupement des Agriculteurs Biologiques 44, les associations CAP44 et Terroirs44, la Fédération Départementale des Civam et Terres de Liens ont été invités à la première réunion publique (entretien avec des membres d'AIRES)

21. MEL, CAR, GABNOR, FRCUMA, AFIP et SAFER

22. L'adjointe au maire de Bouaye parle de « la poule et l'œuf » (entretien, 2015). pour gérer la période d'attente avant exploitation effective $^{23}$. Ensuite, le choix des repreneurs se fait aussi en concertation, avec une participation effective des acteurs concernés, dont les propriétaires au travers de l'AFA, ce qui s'avère un vrai point positif pour les membres d'AIRES.

Portée par un modèle de fonctionnement imposant mais malléable (Nantes) ou basée sur l'interconnaissance (Lille), une gouvernance foncière s'affirme (Bertrand, 2013), non sans répercussions possibles sur la forme de l'agriculture locale.

\section{Donner à lire quelle agriculture ?}

La finalité des démarches est toujours reliée à « l'agriculture », mais l'irruption dans le débat d'autres acteurs que ceux de la sphère agricole insuffle une autre lecture de l'activité, avec de nouvelles exigences en termes de mode de production notamment.

Sur Lille, l'asymétrie est notable entre la démarche suivie dans le cadre des parcs périurbains (accompagnement des stratégies de diversification sans exigence autre que le référencement) et les conditions imposées aux candidats à l'installation sur la zone de Wavrin. Pour la métropole de Lille et l'association Terres de Liens, l'AB était incontournable, à la différence de la CAgr. qui refusait d'écarter des candidats conventionnels. La résolution de ce point d'achoppement est passée par la certification environnementale de niveau 3.

Sur Nantes, dessein environnemental et dessein alimentaire sont souvent conjugués, mais sans recours systématique à la certification $\mathrm{AB}$. NM et la CAgr. ne l'imposent pas, mais certains maires, voire les propriétaires, la plébiscitent volontiers. Par contre, ils portent un intérêt certain aux nombreuses zones de marais qui peuvent venir conforter des pratiques extensives.

La mise en vitrine d'un foncier doté d'attributs positifs pour la ville modifie sensiblement les règles de fonctionnement du monde agricole qui doit répondre à la projection idéelle qu'ont les urbains en particulier, non sans disjonctions ou discontinuités cependant.

23. Difficulté de la concordance dans le temps entre l'accord des élus, des propriétaires et le porteur de projet. Souvent il faut au minimum 1 an et demi. La stratégie est d'avoir des terres disponibles pour des projets mûrs. 


\section{Disjonction et discontinuité : reflet du jeu d'acteurs}

La construction de projets de territoire est rarement linéaire : ce constat vaut pour nos deux terrains, mais de manière distincte.

Pour Wavrin, le lieu même est en gestation depuis 1998 et se place dans la droite ligne de projets antérieurs, notamment de "Maraîchage en Weppes », véritable projet de filière, avec la marque territoriale « Maraîchers des campagnes lilloises ». La définition de la «zone d'activité » résulte ainsi de son rattachement à la direction du Développement économique de l'EPCI, à la différence des parcs périurbains dont l'initiative relève du syndicat mixte ENLM. Dans ces parcs, peu d'exploitants ont finalement adhéré aux programmes proposés (Rouget, 2014), et même dans un autre registre, les projets d'aménagement (cheminements séparés sur le site des Périseaux - Chantier Nature, 1999) n'ont été que très tardivement conduits à leur terme. Alors que dans les années 2000 s'affirme une fonction nourricière, de nouvelles prérogatives sont attribuées au foncier agricole, dissociées spatialement sur Wavrin : pas de vente directe sur place, mais insertion dans des réseaux existants (circuits courts, magasin bio... extérieurs au site). Nous avons sur Lille deux projets parallèles donc, mais un même Vice-Président délégué à l'Espace rural et au monde agricole qui, depuis 2015 et le passage au statut de Métropole, dispose d'un service spécifique.

Sur Nantes, la problématique est différente dans la mesure où la politique est déployée dans 24 communes diversement impactées par les friches, alors que l'intervention est micro-locale (dans les îlots de culture, des parcelles peuvent ne pas dépasser $1000 \mathrm{~m}^{2}$ ). A l'initiative, NM escompte une implication dans toutes les communes, ce qui suppose l'adhésion de tous les acteurs, non sans difficulté parfois (entretien avec le chargé de mission de $\mathrm{NM}$ ). Au démarrage, des réticences étaient inévitables de la part de propriétaires fonciers ${ }^{24}$ ou de propriétaires de chevaux de loisirs. Pour autant, aucun blocage avéré n'a été constaté, et même un dialogue constructif a été noué dans un contexte plus consensuel à partir du moment où ont pu être constatés de premiers résultats de valorisation de

24. «Ils doivent faire leur deuil » selon l'expression d'un élu ; «tuer le rêve » pour l'adjointe à Bouaye. friches. Même, "pour certains propriétaires, la terre retrouve une "valeur" non monétaire et va permettre de nourrir les enfants en bio à la cantine scolaire " (maire de Saint-Jean-de-Boiseau)... Perceptible sur Bouaye et La Chapelle-sur-Erdre (réorganisation du parcellaire pour les chevaux autour d'un lycée et d'un centre équestres), ce processus permet d'affirmer qu'on est dans une trajectoire de changement. De même, les élus municipaux, s’ils peuvent être réceptifs face à la déprise viticole (et la déstructuration du paysage qui s'ensuit), ou, en sud Loire, lorsque l'eau et les marais conditionnent le fonctionnement du territoire, sont beaucoup plus réticents lorsque les friches sont déjà anciennes (donc trop coûteuses à défricher, nord Loire notamment). Une discontinuité interne existe donc, qui montre toute la difficulté à promouvoir une même politique à l'échelle intercommunale si les acteurs ne s'en emparent pas, en particulier les maires. Cette politique a cependant fait ses preuves, ce qui explique sa diffusion dans des collectivités voisines confrontées aux mêmes enjeux : au nord, la communauté de communes d'Erdre-et-Gèvres a pris conscience du problème suite à un diagnostic commandé à la CAgr.; dans le Pays du Vignoble nantais au sud-est, la crise viticole a généré une déprise foncière et de multiples arrachages de vignes, qui inquiètent quant à la pérennité du vignoble dans certaines communes.

Et une dernière discontinuité vient de la sémantique. Pour le nouveau VP de NM, le terme de « friches » a une connotation négative. Il préfère parler de délaissés agricoles, terme plus consensuel qui ouvre des perspectives de mutabilité plus larges (entretien, 2016).

\section{Conclusion}

En définitive, que représentent factuellement les deux projets de zone marâichère et horticole d'une part, de reconquête des délaissés agricoles d'autre part? Peu de choses en surfaces, mais ces deux expériences ont une portée symbolique réelle. Les lieux ne valent pas que pour eux-mêmes, mais pour la mise en ouvre de logiques procédurales multiacteurs qui tendent à devenir la norme (Bertrand, 2013). L'appropriation commune des enjeux fonciers agricoles passe en effet par la formulation de préconisations qui pourraient devenir sclérosantes (débat sur l'AB), voire contre-productives si le 
jeu d'acteurs est trop déséquilibré (Poulot, 2014). Malgré une évidente convergence des représentations de ce que doit être l'espace agricole périurbain, la lisibilité du partenariat ville-agriculture est faible, même si sur Nantes, une plaquette sert de support de communication. Cette question de la lisibilité est à corréler à celle de la légitimité d'une part, et du coût financier d'autre part. Même si le procédé se diffuse très largement, la sélection de projets et/ou de candidat (e)s est tributaire de la composition des comités constitués pour l'occasion et non garants de réussite à court, moyen ou long terme. De plus, le budget alloué est vite conséquent : 3 millions d'euros pour la zone de Wavrin (pour 16 mois de travaux, les parcelles étant disponibles à compter de mars 2016). Dans un contexte de réduction budgétaire, on peut s'interroger sur la capacité à dupliquer ce type d'opération.

Les discours des différents acteurs interrogés depuis 2014 s'inscrivent dans le cadre des réflexions conduites autour des projets alimentaires territorialisés de la loi d'avenir pour l'agriculture. Le foncier agricole, appréhendé en tant que ressource territoriale incontournable, devient un maillon d'une filière alimentaire locale dont les enjeux se déportent sur des volets d'acceptabilité sociale, de nutrition/santé, de bien-être, etc. Autant de nouveaux acteurs et de nouvelles manières de le lire en perspective.

\section{Bibliographie}

Banos V., Sabatier B., 2010. Les espaces périurbains non bâtis en France : entre publicisation "urbaine" et privatisation "rurale"?, Articulo, Special issue 3 [http://articulo.revues. $\mathrm{org} / 1524]$.

Banzo M., Couderchet L. 2013. Intégration de l'agriculture aux politiques et projets territoriaux urbains. Le cas bordelais, Sud-Ouest Européen, no 33, p. 5-16.

Bertrand N. (coord.), 2013. Terres agricoles périurbaines. Une gouvernance foncière en construction. Ed. Quæ, coll. Update Sciences et Technologies, $250 \mathrm{p}$.

Bonduelle B., 1992. Lettre aux 86 maires de Lille. Éditions La Voix du Nord, Lille, 245 p.

Bonnefoy S., 2011. La politisation de la question agricole périurbaine en France : points de repère, Urbia, $\mathrm{n}^{\circ} 12$, p. 17-38.

Brand C., Bonnefoy S., 2011 . L'alimentation des sociétés urbaines : une cure de jouvence pour l'agriculture des territoires métropolitains?, VertigO, vol. 11,2, septembre [http:// vertigo.revues.org/11199].
Chantier Nature, 1999. Étude d'aménagement de l'espace naturel intercommunal, Lille, Syndicat de communes de Fâches-Thumesnil, Templemars, Vendeville et Wattignies, Agence de Développement et d'Urbanisme de Lille, 97 p.

Croix N., 1999. La Terre, entre Terroir et Territoire : Mutations foncières et organisation des campagnes armoricaines méridionales, 1968-1998, thèse pour le doctorat d'État de géographie, Université de Nantes, 1142 p.

DARLY S., 2012. Le partage de la terre agricole, entre conflits fonciers et projet collectif (l'exemple des domaines agricoles du Plateau briard), in PAPY F., Nowveaux rapports à la nature dans les campagnes, Paris, Quæ, coll. «Indisciplines », p. 41-53.

DGUHC, 2004. Politiques foncières locales. Prendre en compte le foncier dans le projet de territoire. Direction Générale de l'Urbanisme, de l'Habitat et de la Construction, collection «les outils », 64 p.

Douay N., 2013. La planification urbaine française : théories, normes juridiques et défis pour la pratique. L'Information géographique, vol. 77, n 3, p. 45-70.

Geniaux G., Napoléone C., 2007. La constructibilité anticipée des terres agricoles, Études foncières, n ${ }^{\circ}$ 125, p. 12-14.

Glon E., Pecqueur B., 2006. Développement et territoires : une question d'environnement et de ressources territoriales, Territoires en Mouvement, vol. 1, p. 13-22.

Guéranger D., 2012. La monographie n'est pas une comparaison comme les autres Les études de l'intercommunalité et leur territoire. Terrains E travanx, vol. 2, no 21, p. 23-36.

Le Caro Y., Pierre G., Margetic C., 2010. Le statut social de la terre agricole dans un espace rural en voie d'urbanisation, le Coglais (Bretagne). Intérêt et limites d'enquêtes par groupes de discussion. Aoste, colloque ASRDLFAISRe, Identité, qualité et compétitivité territoriale, 20-22 septembre. [http://asrdlf2010.let.fr/documents/papiers/S5/ LeCaro_176.pdf].

LÉvy J., 2010. La ville est le développement durable. Métropolitiques. [http://www.metropolitiques.eu/La-ville-est-le-developpement.html].

Loudiy S., Bryant C., Gueringer A. (dir.), 2011. Dossier : Acteurs et projets au cœur des agricultures urbaines et périurbaines. VertigO, vol. 11, nº 2 [https://vertigo.revues. org/11071].

Margetic C., 2014. Des espaces naturels pour pérenniser l'activité agricole : exemple de l'EARL Batard à Port-Saint-Père (44). Entretien avec Bertrand Batard, Revue POUR, n 24, p. 267-273.

Margetic C., Chaucesse J.N., 2012. La politique de « reconquête des friches agricoles » dans l'agglomération nantaise, Les Cahiers Nantais, n ${ }^{\circ}$ 2, p. 59-68.

Margetic C., Noël J., Dufeu Y., Le Grel L., 2016. Organisations collectives territorialisées. Le département comme référence, in Mundler P., Rouchier J., Alimentation et proximités : jeux d'acteurs et territoires, Educagri, p. 131-149.

Novarina G., 2003. Ville diffuse et système du vert, Revue de Géographie Alpine, vol. 91, nº 4, p 9-17.

Pecqueur B., 2006. Le tournant territorial de l'économie globale, Espaces et sociétés, vol. 2, n 124-125, p. 17-32. 
Pecqueur B., Lajarge R., Lardon S., Loudiy S., 2012. Ressources territoriales - Politiques publiques et gouvernance, Rapport scientifique final, RessTerr Rhône-Alpes \& RessTerr Auvergne, $43 \mathrm{p}$.

Poulot M., 2013. Du vert dans le périurbain, EspacesTemps.net [http://www.espacestemps.net/articles/du-vert-dans-le-periurbain].

Poulot M., 2014. Agriculture et acteurs agricoles dans les mailles des territoires de gouvernance urbaine : nouvelle agriculture, nouveaux métiers?, Espaces et sociétés, vol. 3, $\mathrm{n}^{\circ} 158$, p. 13-30.

Poulot M., Rouyres T., 2007. Refaire campagne en Île-deFrance, Norois, $n^{\circ} 202$, p. 61-71.

Rouget N., 2008. Les dynamiques agricoles dans les espaces urbains et périurbains. Diversification et stratégies d'adaptation des agricultures. Les cas des périphéries sud-est de Lille et Nord de Lens, Thèse de doctorat, Université de Paris Ouest - Nanterre-La Défense, 400 p.

Rouget N., 2012. La gestion des espaces ouverts de Lille Métropole. Produire un « paysage vivant », mettre à contribution l'agriculture, Projets de paysage. [http://www.projetsdepaysage. fr/fr/la_gestion_des_espaces_ouverts_de_lille_metropole].
Rouget N. 2014. Nature urbaine et agriculture dans le NordPas-de-Calais. Synergies et paradoxes, in Donadieu P. (dir.), L'Agriurbanisation : rêves ou réalités?, Paris, Editopics, p. 56-73.

Schmiтt G., 2007. Inégalités écologiques et utilisation du sol : situation de la région Nord-Pas-de-Calais, Développement durable et territoires, Dossier 9 [http://developpementdurable. revues.org/3472].

Sснмітт G., 2009. Valeurs et usages de l'espace : approches méthodologiques des dynamiques foncières dans la région Nord-Pas-de-Calais. Thèse de doctorat en Géographie, aménagement de l'espace et urbanisme, Université de Lille 1, $491 \mathrm{p}$.

Serrano J., 2012. L'insertion de l'agriculture dans les projets d'aménagement des élus urbains, Environnement Urbain / Urban Environment, vol. 6, p. 32-49 [http://id.erudit.org/ iderudit/1013711ar].

Vidal R., Fleury A., 2009. La place de l'agriculture dans la métropole verte, Projets de paysage, [http://www.projetsdepaysage.fr/fr/la_place_de_l_agriculture_dans_la_metropole_ verte\#citation]. 


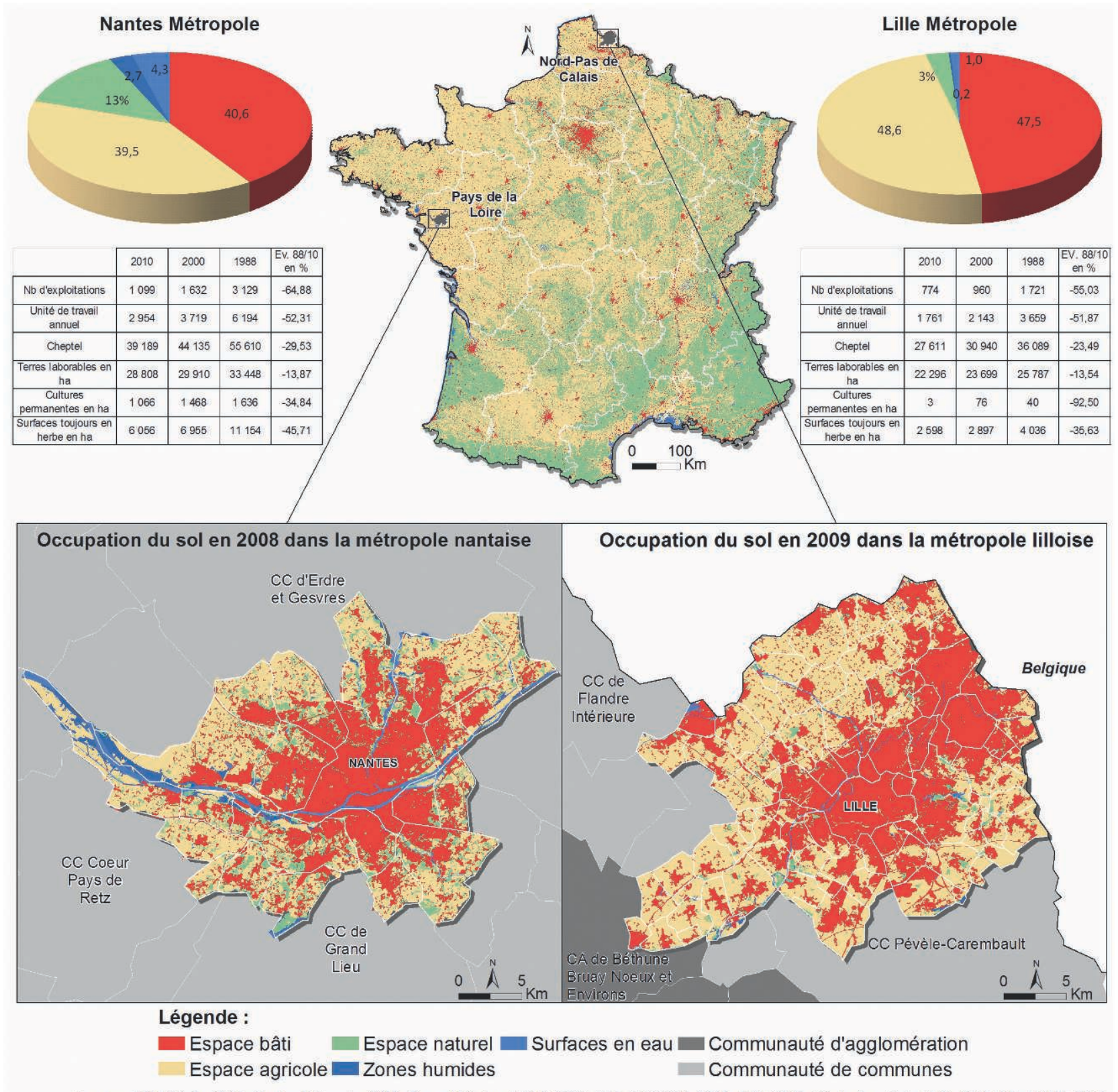

Sources : IGN, Géofla, 2013; Nantes Métropole, 2012 ; Conseil Régional NPdC, 2012, AEE, CLC-2006, 2012 ; RGA, 2010. Réalisation : Schmitt G., CUE-LNF - UVHC, 2014.

Figure 1 : Les deux métropoles étudiées

Two case studies: the Lille and Nantes metropolitan areas 
Planche II (Christine MARGETIC et al. - Le foncier agricole à l'épreuve de la multifonctionnalité...)

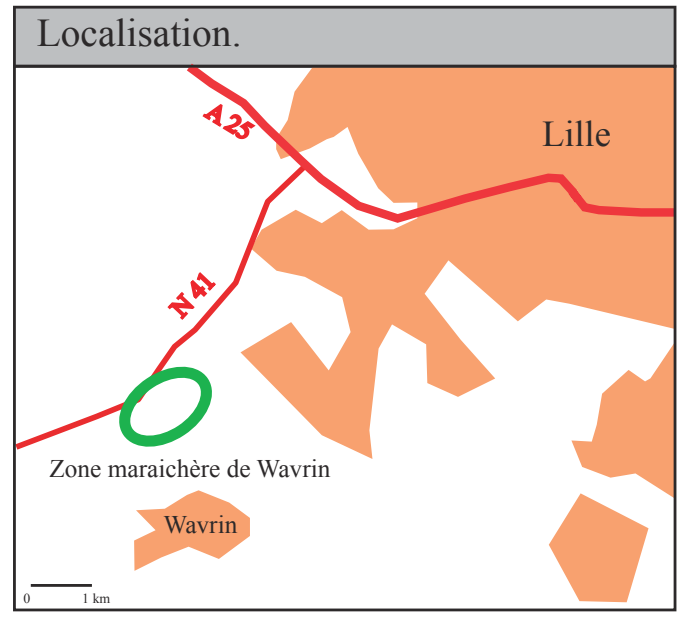

Figure 2 : La zone maraîchère et horticole de Wavrin Wavrin: a new market-gardening and horticultural zone

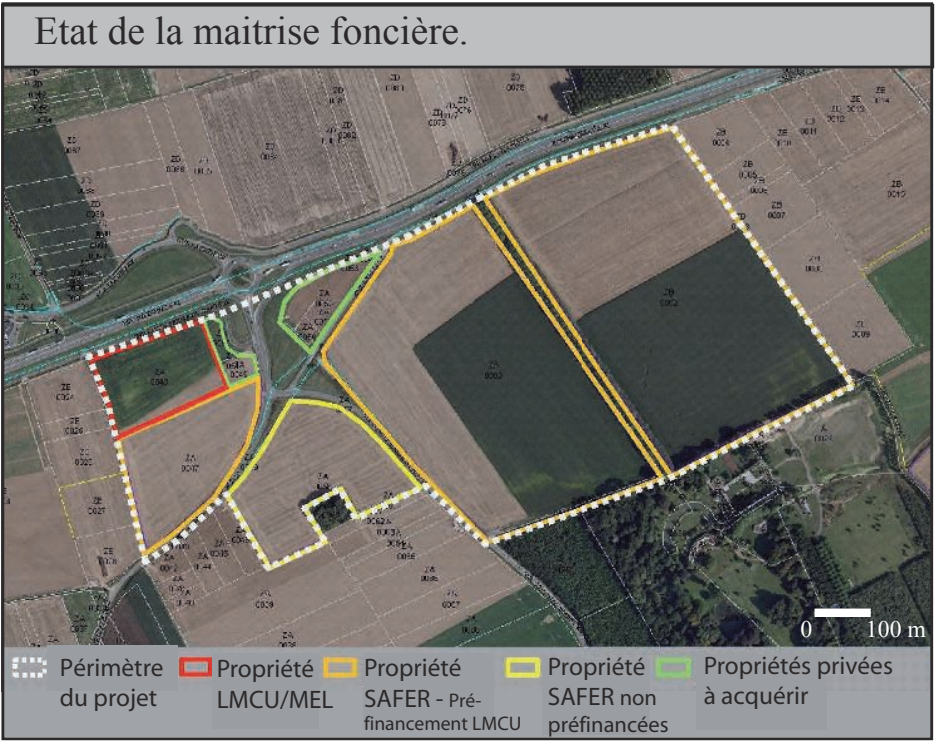

Lille Métropole, Direction espace naturel et urbain.

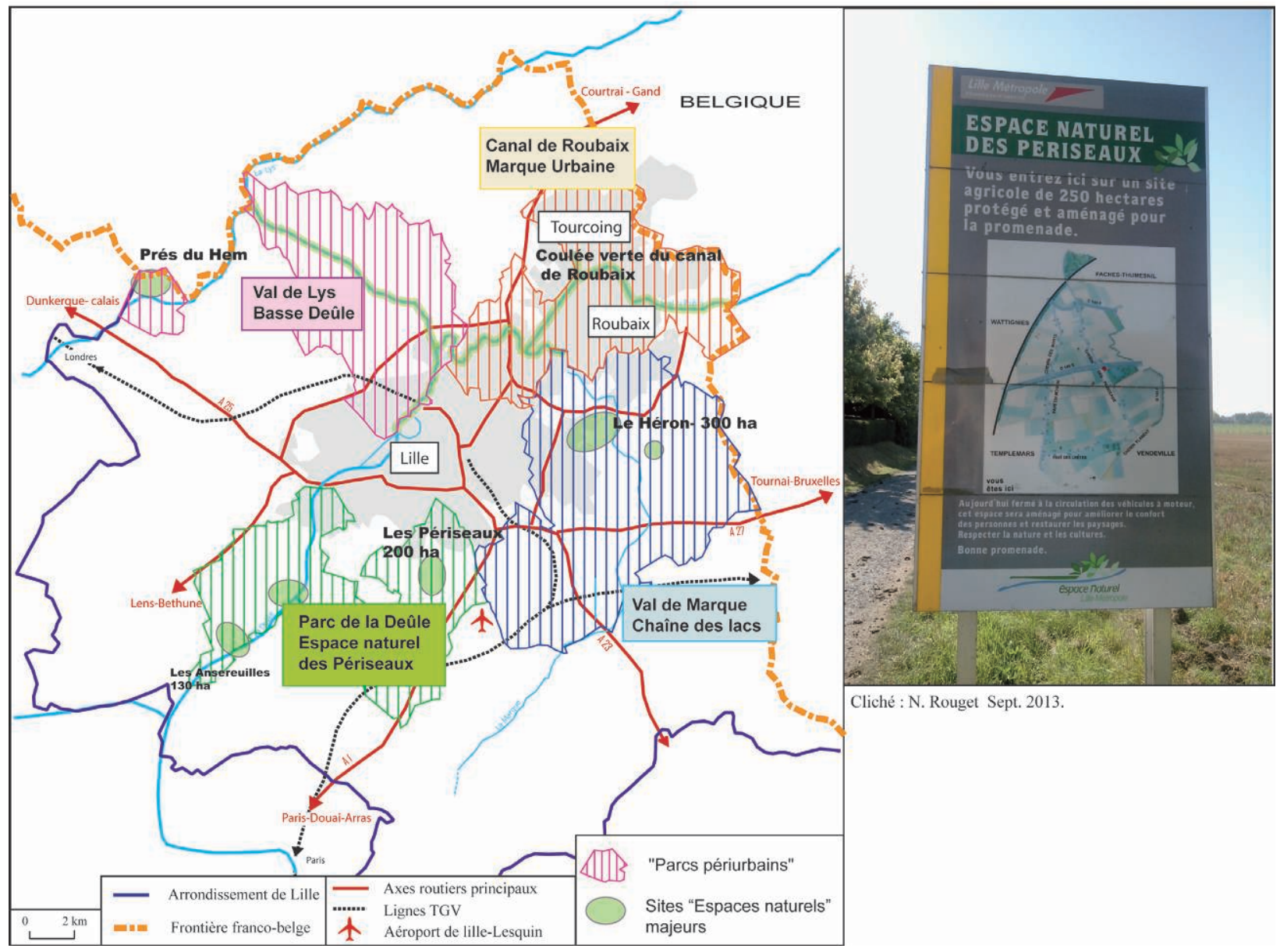

Figure 3 : Les « parcs périurbains » lillois

"Agroparks" in the Lille metropolitan area 


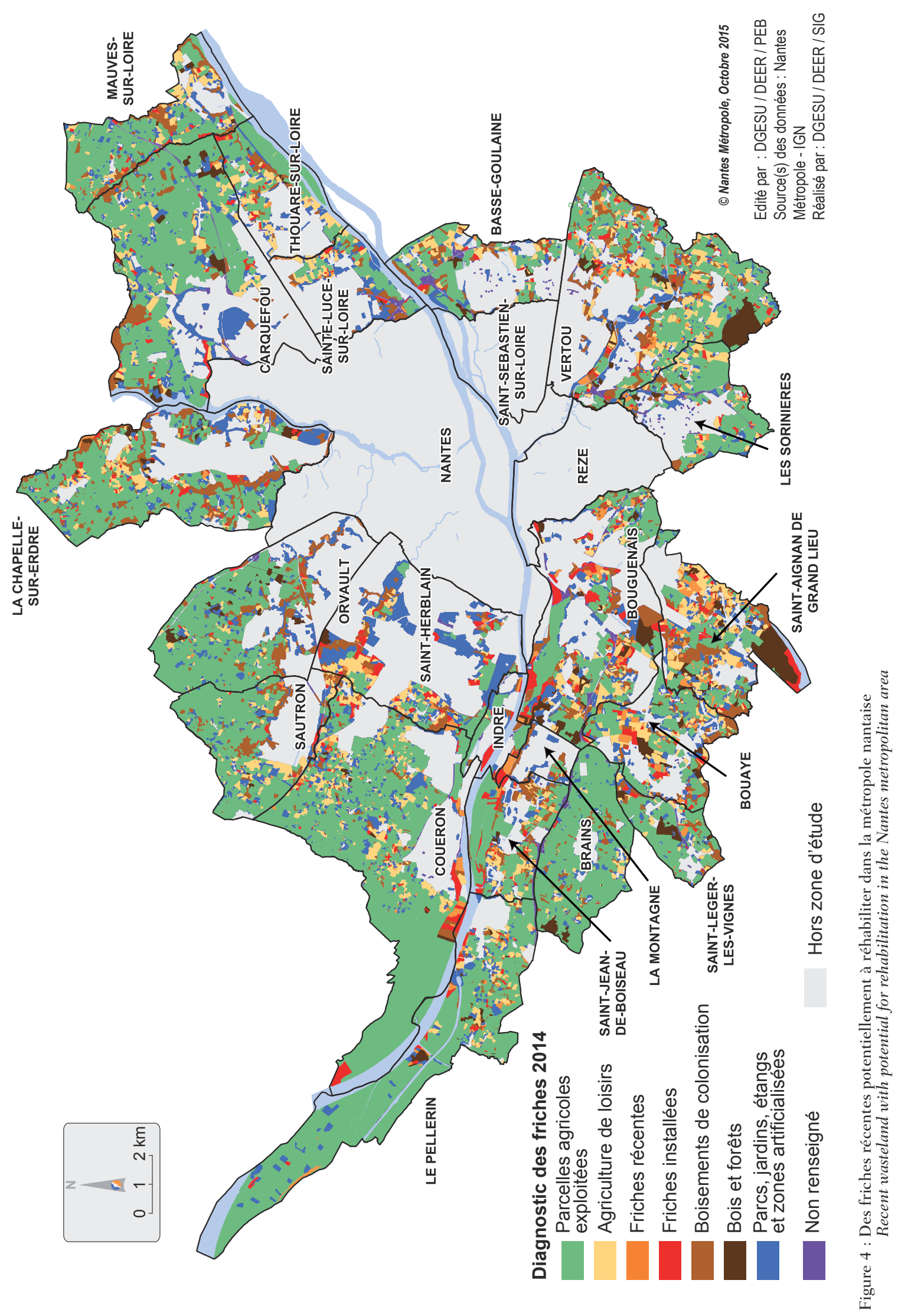




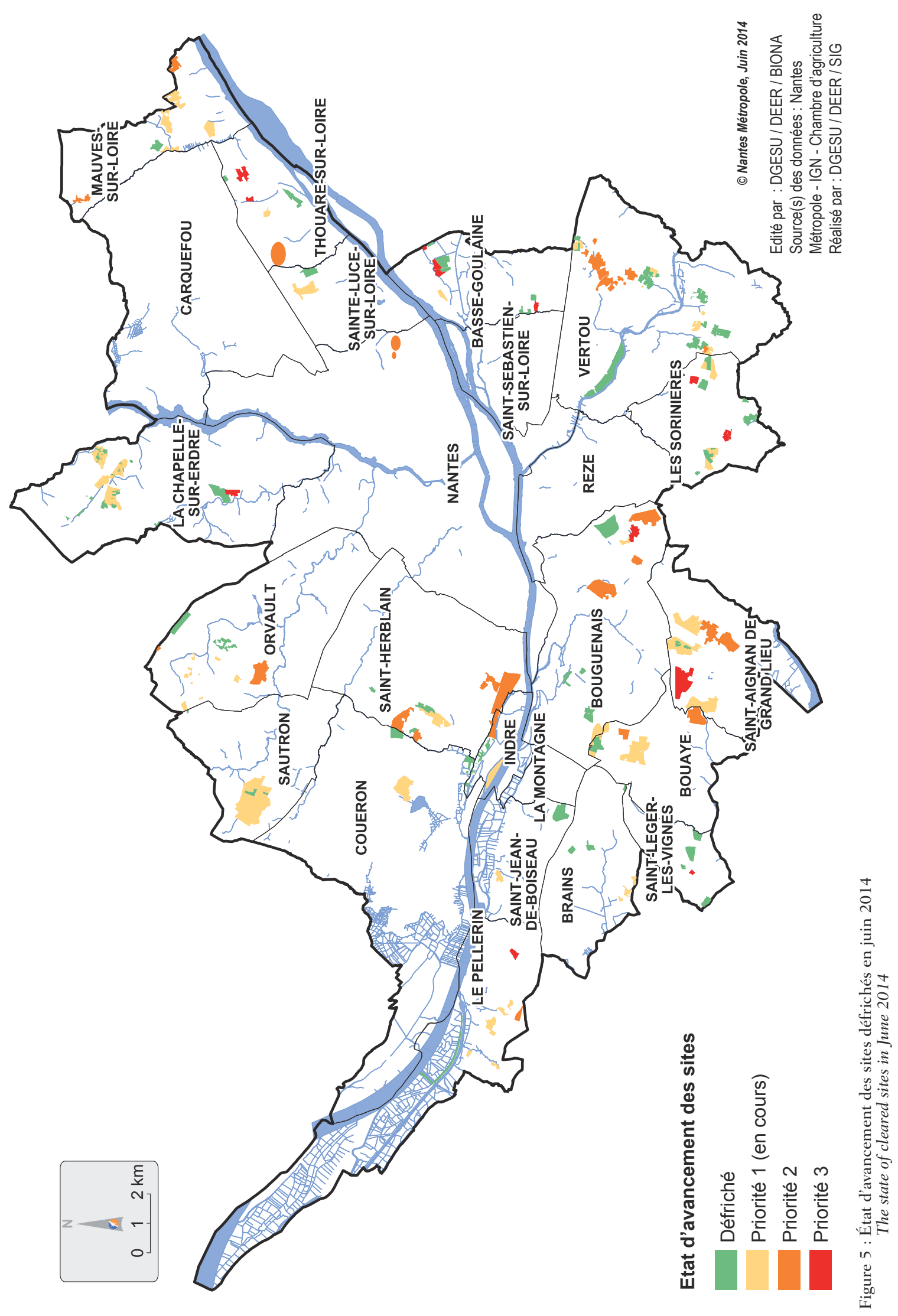

